\title{
La expresión artística: Otro desafío para la educación rural
}

\author{
Artistic Expression: Another Challenge for Rural Education
}

\author{
Sonia Álvarez Castro \\ Centro Educativo San Felipe \\ Santo Domingo de Heredia \\ Costa Rica \\ sonielena29@yahoo.com \\ María del Carmen Domínguez Lacayo² \\ Ministerio de Educación Pública \\ Escuela Montealegre \\ El Amparo de Los Chiles \\ Alajuela, Costa Rica \\ soledad08amor@hotmail.com
}

Recibido 10 de junio de 2012 • Corregido 08 de octubre de 2012 • Aceptado 07 de noviembre de 2012

Resumen. Este ensayo establece parámetros sobre la necesidad de buscar espacios que fortalezcan el tejido social y promuevan el desarrollo integral, a través de la expresión artística como metodología de expresión de sentires y construcción de las identidades tanto culturales y sociales como individuales, las cuales en nuestra coyuntura globalizada, han sido desgastadas mediante la homogenización de las vivencias.

Palabras claves. Expresión artística, construcción de las identidades, educación para un desarrollo integral.

Abstract. This paper identifies the parameters required to create opportunities that would strengthen the social fabric and would promote a comprehensive development through the artistic expression as a method for expressing feelings and constructing -cultural and social-identities as individuals, which, in our global context have been eroded by the homogenization of experiences.

Keywords. Artistic expression, identity construction, education for a comprehensive development.

\footnotetext{
1 Licenciada en Educación con énfasis en Educación Rural. Labora en Santo Domingo de Heredia, Costa Rica. Estudiante de la Maestría en Educación Rural Centroamericana.

2 Licenciada en Educación con énfasis en Educación Rural. Laborara en Montealegre de Los Chiles, Costa Rica. Egresada de la Maestría en Educación Rural Centroamericana.
} 
La globalización ha acelerado una dinámica de relaciones inter comerciales, inter sociales e inter culturales, existente y permanente entre los pueblos. Las tecnologías de la información y comunicación contribuyen a que la adopción, absorción e incluso la imposición de elementos culturales universalicen, de alguna manera, la expresión artística, en el sentido de comprensión y expresión de la comunicación. Durante siglos, el ser humano ha utilizado la expresión artística desde la perspectiva del gozo y del disfrute, sin amalgamar su importancia para construir desarrollo humano y fortalecer el tejido social. "(...) centró en la difusión cultural, esto es, en llevar a la producción y educación estética al pueblo desde diferentes medios y modalidades (...)" (Mariscal, 2009 p. 20).

(...) La politización de la esfera cultural, la creciente economización de sus dominios, y la progresiva profesionalización de sus agentes, han incrementado la impronta de creaciones realizadas por especialistas y de elaboraciones simbólicas desterritorializadas o surgidas en espacios distantes (...). (Bayardo, 2009, pp. 11-12)

Las políticas globalizantes de la cultura incurren de manera constante y afectativa en la construcción de las identidades. Los gestores culturales interfieren en la renovación de aprendizajes y afectan, con ello, la "(...) conformación de las subjetividades y los cotidianos; sobre la definición de agendas (...) públicas; y sobre la constitución de actores sociales (...)" (Bayardo, 2009, p. 12). Se forjan, entonces, diversas expresiones artísticas: unas encargadas de homogenizar a la cultura, de imponer supremacía y otras intentan, a arañazos, reivindicar la peculiaridad local. Esta singular guerra de lo local a lo global y viceversa genera la necesidad de voltear miradas para rediseñar y configurar la cultura en lo referente a la expresión de las identidades, la expresión de la nacionalidad, así como la convivencia en la diversidad y para esta, en aras de construir una sociedad más responsable con base en conocimientos artísticos, culturales, antropológicos, éticos y estéticos.

Una manera de establecer arraigo cultural y empatía por las culturas autóctonas y hegemónicas podría darse desde la educación artística y cultural, utilizando métodos dialógicos y creativos que fomenten, en docentes y dicentes, ambientes de autoaprendizaje propicios para la configuración de las identidades, a través de prácticas pedagógicas que desarrollen sensibilidad, autonomía y pensamiento crítico.

La educación como gestora de sociedad para las sociedades tiene hoy por hoy un mayor compromiso: repensar la forma, el cómo, el para qué educar, pues con el devenir de los tiempos, se han viciado algunos procesos, y se ha perdido confianza en la educación. Desde una perspectiva antropológica se define la educación como: “(...) un mecanismo de reproducción social, que no sólo se presenta como un proceso de endoculturación de las prácticas culturales (Herkovitz, 2004), sino también (...) de las relaciones de dominación y desigualdad de una sociedad" (Borurdieu y Passeron, 2003, en Mariscal 2009, p. 17). Las empresas trasnacionales, 
con el afán de crecimiento económico y expansión de bienes, manipulan y esclavizan en nombre de la educación. Por esta razón nos vemos en la obligación de buscar formas de rescatar o redescubrir, en la educación, el aliciente; el medio para reorientar el desarrollo, que permita progreso para todos en iguales condiciones sin distingos de etnias, clases sociales, edad ni género; un motor de cambios que genere progreso y no necesidades.

En la búsqueda de sociedades más equitativas e integrales, la educación artística podría establecer, desde otras perspectivas, espacios para la construcción de competencias que permitan a niñas, niños, jóvenes, adultas y adultos aprender a aprender, aprender a convivir y aprender a ser, potenciando la creatividad, el autoestima y el trabajo en equipo. Marchessi (2006), en Educación Artística, cultura y ciudadanía, establece que: "(...) el desarrollo de la capacidad creativa, la autoestima, la disposición para aprender, la capacidad de trabajar en equipo o el pensamiento abstracto encuentran en la educación artística una estrategia potente para lograrlo" (p. 7).

El arte es una herramienta de desarrollo interpersonal, de expresión de ideas y sentimientos, de promoción de cambios sociales y culturales. Como herramienta, facilita el autoconocimiento y la autovaloración, ya que crea percepciones más amplias y analíticas de la realidad. Según Abad (2006) "(...) las artes se han transformado (...) manteniendo siempre abierto el debate de su relación con la realidad y reelaborando sus propios códigos de representación (...)" (p. 17). Vista la educación artística desde una función social, permite sensibilizar a la persona, estimulando la intuición y la creatividad, promoviendo la seguridad y la autoconfianza por medio de la expresión artística. “(...) La educación artística puede y debe ofrecer ámbitos de (...) reflexión y compromiso, de manera individual y colectiva, que se proyectan en la búsqueda de una mayor calidad en la relación entre arte y vida" (Abad, 2006, p. 17).

Marchessi (2006) propone la construcción de ciudadanías interculturales y la apropiación de las identidades desde la educación artística: "Conocer, valorar y disfrutar de las expresiones artísticas (...) fomenta (...) el reconocimiento y el respeto de la diversidad cultural y personal" (p. 8). La educación artística como herramienta, posibilita el desarrollo social y la afectividad, ya que promueve inclusión, y permite plantear acciones de transformación personal y social que contribuyen con el desarrollo del tejido social y la convivencia armoniosa. Además, se convierte en competencia facilitadora de habilidades expresivas y comunicativas; estimula relaciones equitativas dentro de heterogeneidad, al mismo tiempo que busca un equilibrio en las relaciones en la diversidad y para esta. "(...) El arte como experiencia liberadora ha de contribuir a canalizar la energía social y a crear nuevas formas de comunicación intercultural, de convivencia y de cambio social (...)" (Jiménez, Aguirre y Pimentel, 2006, p. 11).

Las artes ofrecen modos únicos del conocimiento y de la percepción social e individual a través de códigos testimoniales de la cultura y forma de vida que reflejan la cotidianidad y promueven una conciencia crítica de las realidades; frente a los mecanismos excluyentes, propician las construcciones de ciudadanías interculturales, sustentables y responsables. "(...) Las funciones actuales de las artes son presentadas como una apertura de opciones y 
URL: http://www.una.ac.cr/educare

posibilidades que deben atender a las diferentes perspectivas y necesidades de cada persona y colectivo de referencia, sirviendo (...) para construir la realidad en sus propios términos (...)" (Abad, 2006 , p. 17).

Efland, citado por Abad (2006), establece la función de las artes desde una perspectiva de construcción histórica de las identidades y realidades que enmarcan la adopción y construcción de aspectos culturales determinantes.

La función de las artes a través de la historia cultural humana ha sido y continúa siendo una tarea de 'construcción de la realidad'. Las diferentes artes construyen representaciones del mundo [...] que pueden inspirar a los seres humanos para comprender mejor el presente y crear alternativas de futuro. Las construcciones sociales que encontramos en las artes contienen representaciones de estas realidades sociales que contribuyen a la comprensión del paisaje social y cultural en el que habita cada individuo (Efland, 2004, p. 229). (Abad, 2006, p. 19)

La presencia del arte en la educación, a través de la educación artística y de la educación por el arte, asiste al desarrollo integral y pleno de las niñas, de los niños, de las jóvenes y los jóvenes, de las adultas y los adultos. ¿Cómo privar, entonces, a las comunidades rurales de esta opción, facilitadora y promotora de desarrollo humanístico?

El desarrollo, visto como proceso educativo desde lo humano y para lo humano, propone progreso, entendido este como la satisfacción de las necesidades de las comunidades y su población. La educación como acto de construir aprendizajes incluye el reconocimiento de su cultura, la comunicación de sentires y pensares desde perspectivas antropológicas y etnográficas, por lo tanto, constituye un acto de recreación y reconstrucción de las realidades e identidades. Freire, citado por Carmen (2004), establece la educación como: "(...) la búsqueda permanente de la gente, unos con otros, para llegar a ser más plenamente humano, en el mundo en que existen (Freire, 1973, p. 94, citado en Carmen, 2004, p. 89). Desde esta perspectiva, la educación como herramienta problematizadora da propiedad y control en la creación de conocimientos que generarán, a la postre, capacidades para el desarrollo.

"Hablar de educación para el desarrollo humano significa tener en cuenta varios aspectos: superar las miradas sicologistas y fragmentadas por etapas del desarrollo; trabajar por el afianzamiento de la identidad, la autonomía y la creatividad (...)" (Castro, 2007, p. 167). La educación a través del arte proporciona una serie de fortalezas en los individuos, al aportarles valores morales y espirituales y al beneficiar, así, el entramado social en las zonas rurales. Se logra, de esta forma, una mejor inserción en el desarrollo global, ya que el arte permite desplegar, en las personas, destrezas y habilidades tanto físicas como mentales y espirituales, les abre horizontes de valoración propia y del entorno y aporta a la sociedad individuos capaces de amar su naturaleza y preservarla. 
La escuela rural, que siempre ha estado involucrada y comprometida con el desarrollo de las sociedades y con el crecimiento integral de los individuos, esperanzadoramente necesita abrir las puertas a tendencias pedagógicas, estructurales y analíticas que permitan, a sus comunitarios y comunitarias, nuevas formas de vida. "(...) Una de las tareas del educador - la educadora progresista, a través del análisis político serio y correcto, es descubrir las posibilidades -cualquiera que sean los obtáculos- (...)" (Freire, 1993, p. 25).

Gaskins y Elliot, citado por Mollins, Castillo y Silber (2000, p. 16) establecieron que "(...) Aprender exige el uso de diversas habilidades y estrategias de procesamiento (...)". A partir de este planteamiento, concebimos la adquisición de saberes y la apropiación de culturas entre los pueblos rurales desde la expresión artística como un medio de aprendizaje para, con ella, profundizar la construcción de estrategias cognitivas con la niñez, la juventud y las personas adultas, ya que cada sujeto aprehendiente es protagonista de su propio proceso de aprendizaje.

La educación artística es expresividad, es representación de sentires y pensares desde el ser y propicia representaciones reales de la cotidianidad. Salvando al lector de imaginarios sobre el crear artistas académicos, es que las autoras dirigimos las miradas al arte a través de la expresión artística. Desde esta perspectiva, se define la expresión artística como una herramienta ideológica, corporal, instrumental y comunicativa de la expresión de sentimientos, deseos y conceptos. Baczko, mencionado por Lagos, González y Cárdenas (2009), define la expresión como "(...) parte del imaginario social de un grupo humano. A través de estos imaginarios sociales, una colectividad designan [sic] su identidad, elaborando una representación de sí misma (...)" ( p. 26).

Podría la educación gestionar, en la expresión artística, la esperanza de la formación integral para el desarrollo sostenible y sustentable que beneficie al ser humano y su entorno. "(...) La educación y el arte por sí mismos generan la evolución social a partir del desarrollo del pensamiento y la sensibilidad (Azuela, 2005 citada por Mariscal p. 20, 2009). A través de disciplinas artísticas, el ser humano puede canalizar ideas y sensaciones intelectuales hacia el exterior y hacia los demás, de suerte que se desarrollan en los individuos diversas capacidades que les benefician en la convivencia con sus semejantes.

Por lo tanto, la expresión artística desarrollada por la comunidad y para la comunidad se convierte en una oportunidad de articulación para el conocimiento y la transferencia de las culturas; establece la confluencia de otras identidades, favoreciendo la convivencia pacífica y el diálogo desde la interculturalidad; al mismo tiempo, promueve la construcción de espacios de madurez emocional y de auto aceptación, y permite un desarrollo equilibrado.

Además, permite el logro de la autocomprensión y la aceptación de la otredad, actitudes necesarias para el buen vivir en este mundo de trasformaciones constantes. Este hecho ha estimulado un desarrollo en forma continua y la necesidad de nuevas estrategias que transformen las políticas globalizantes y sobreponga, en sus mesas, nuevas filosofías de vida que posibiliten cambios radicales de convivencia. Según Jiménez et al. (2006), la educación desde la expresión artística "(...) se convierte en uno de los sectores de mayor importancia 
en la posibilidad de crear valores, habilidades y competencias para la autoexpresión y la comunicación, para fomentar nuevas formas de convivencia (...)" (p. 11).

Las economías globalizantes vendedoras de imágenes, de colores, de sonidos, de ritmos -consumistas imperantes de una cultura hegemónica- han debilitado las construcciones populares y culturales de las identidades, incluyendo ópticas propias y accesibilidad. Además, han promovido la exclusión desde lo autóctono. Por eso, la necesidad de fortalecer, desde la educación, las identidades para desarrollar, en las personas comunitarias, sentido de pertenencia. Dichos procesos socioeconómicos han modificado los espacios de culturalización: las personas, gracias a los medios de comunicación, mantienen relaciones comunicativas con facilidad, lo cual permite construir cercanías desde lo virtual. Torres y Marrow, citados por Castro (2007), exponen que la globalización "(...) elimina las fronteras nacionales y afecta profundamente en la construcción de las identidades nacionales (...)" (p. 159).

Cuando Paulo Freire habla de la alfabetización, como la lectura del mundo, permite idear o construir nuevas formas para aprender a leer y a escribir, vistas desde todas las aristas posibles e imaginables. "(...) Aprender a leer y escribir es un proceso de comunicación a través de culturas (...)" (Carmen, 2004, p. 136). Como proceso de culturalización, implica expresión de sentires y saberes desde una gestión cultural artística, proyectada a la edificación de desarrollo integral. Piñón (2005), secretario de la Organización de Estados Iberoamericanos, en el informe elaborado por la red iberoamericana de centros y unidades de formación en gestión cultural, expresa: "La gestión cultural es sin duda un campo emergente, con una fuerza dinámica, tanto desde la óptica favorecedora de la cohesión y el desarrollo de las sociedades (...)" (p. 11).

Bass, mencionado por Rivas (2008), señala que la cultura “(...) tiene que ver con cohesión social, con identificación con las formas de gobierno, con expresiones culturales y comportamientos sociales que hacen a la sociedad más cohesiva y compleja, y es asumida como la suma de individuos (...)" (p. 2). Por esta razón, desde la concepción de desarrollo para la humanidad y por ella, la educación por el arte o a través de la expresión del arte proyecta la cultura y la locución de esta como herramienta para la apropiación de las identidades y el fortalecimiento del tejido social. Se valora, así, la expresión de la cultura y se la reconoce como vínculo de superación y expansión de la creatividad. "[La cultura], en sí misma, es un ejercicio que incentiva la participación, la integración, la democratización (...) y la multiplicación del arte en los entramados en que habita" (Rivas, 2008, p. 6).

Históricamente la expresión de cultura, de sentires y saberes estaba enraizada a la oralidad, al canto, a la danza, a la actuación o representación de tradiciones; sin embargo, la globalización ha generado procesos de transformación y aculturalización: "(...) ya no existe una sociedad, por remota que sea, que no haya sido afectada, forzada o invadida, directa o indirectamente, por la sociedad industrial (...)" (Carmen, 2004, p. 137).

En Kenia, El Centro Comunal Educativo y Cultural inició en 1980 un proyecto con mujeres, en el cual utilizaron el teatro como herramienta alfabetizadora y lograron, con éxito, no solo la aprendiencia de codificar las palabras habladas en escritas, sino de trasmitir sus sentimientos, 
los de todo un pueblo, con ansias de ser escuchado, tanto así que la policía prohibió el teatro que la gente (mujeres y hombres) había construido con sus propias manos y sudor. Una de las participantes concluyó que "(...) el régimen puede destruir los centros de la gente e incluso abolir el teatro: ¿pero pueden abolir a la gente? (...) (Ngũgĩ wa Thiong'o, 1982, citado en Carmen, 2004, p. 145). Con esto se recalca que la expresión artística es una de las formas de construir identidades y de trasmitir sentimientos -los cuales pueden ser prohibidos, pero las personas siempre buscarán cómo comunicarlos-.

Volviendo al caso de las mujeres kenianas, ellas aprovecharon los espacios de actuación no solo como medio de codificación de escritura, sino también como herramienta de desarrollo, como práctica transformadora autónoma y concientizadora, desde sus necesidades y potencialidades. Para Kidd, mencionado por Carmen (2004), el teatro, en general las artes, vistas desde las capacidades de expresión, pueden convertirse en trasmisores de aprendizajes y agentes de desarrollo mediante la autoexpresión y apropiación identitaria:

Existe un interés creciente en el teatro como herramienta de desarrollo. Este interés va desde (a) quienes ven el teatro como un medio más efectivo para transmitir información y persuadir a la gente para que cambie sus prácticas y actitudes. Y (b) quienes consideran al teatro como un proceso de concientización y organización controlado por los participantes [cursivas añadidas], mediante el que los pobres pueden transformar [cursivas añadidas] las estructuras que les mantienen explotados y dependientes (Kidd, 1982, p. 7). (Carmen, 2004, p. 146)

Al hablar de fortalecimiento de las identidades a través de la expresión artística, no podemos dejar de mencionar el trabajo realizado por Friedl Dicker-Brandeis, vienesa que trabajó en campos de concentración durante la Segunda Guerra Mundial, quien, utilizando la expresión artística, intentó fortalecer las identidades y "(...) liberar a los menores de tensiones y miedos, al mismo tiempo que les ofrecía una ventana para la imaginación y la esperanza" (Reina y Romero, 2009 p. 1).

La expresión del arte a través de prácticas artísticas no es simplemente otra estratagema desmistificadora y empoderadora, basada en el principio de que si se puede actuar, cantar, bailar, escribir o recitar escenas construidas desde los saberes de las pobladoras y los pobladores, también se puede, en la vida real, apropiarse y responsabilizarse de un desarrollo autónomo sustentable y humanístico. Además, la expresión artística puede ser analizada como medio de comunicación, el cual, a diferencia de los medios de comunicación de masas, globalizantes y hegemónicos, se construye desde sus participantes, de forma equitativa. “(...) Están (...) en manos de la población misma, y se utilizan para proyectos de pequeña escala, son desarrollistas y no alienantes (...) (Leis, 1980, p. 16; Kees Epskap, 1989, p. 94)" (Carmen, 2004, p. 147). 
Para la educación rural, debe ser imprescindible construir espacios de aprendizajes creativos, transformadores e innovadores que garanticen, en la niñez, en la juventud y en las personas adultas, un mañana esperanzador y prometedor, que garantice desarrollo cultural, humano y sustentable. "(...) La nueva pedagogía crítica, renovada y profundamente humanista, [sic] debe considerar que constituye como nunca antes un instrumento para el cambio social (...)" (Brito, 2008, p. 17). "(...) El desarrollo rural significa la gente humanizando y recreando el paisaje" (Carmen, 2004, p. 11).

Abad (2006), por su parte, propone una educación artística, como puerta de acceso a la construcción de sociedades equitativas, solidarias con apropiación de sus identidades culturales, individuales y colectivas, "(...) que proporcione los instrumentos y las competencias para establecer vínculos de pertenencia a una cultura como propuesta activa de desarrollo humano (...)" (p. 21).

La cultura le ha adjudicado, a las artes, la función de representar y comunicar valores y visiones de la experiencia humana, "(...) manteniendo siempre abierto el debate de su relación con la realidad y reelaborando sus propios códigos de representación a través de símbolos e instrumentos culturales" (Abad, 2006, p. 17). De esta forma, en el ámbito latinoamericano se han desarrollado proyectos como el realizado en el municipio de Embú Sao Paulo, Brasil, en donde las autoras validan el teatro como estrategia para la construcción de la paz y la cimentación de sociedades sin violencia. “... [Esta es una estrategia] de movilización y empoderamiento de los actores (...) para la promoción de la salud, enfrentamiento y reducción de la violencia (...)" (Harada, Pedroso y Pereira, 2010, p. 423).

Harada et al. (2010) son ejemplo de la utilización de herramientas de expresión artística que promueven la apropiación de identidad cultural mediante la vinculación de temas de salud y educación en la solución de fracturas y conflictos sociales al articular las transversalidades político sociales como enfoque para el desarrollo.

Carmen (2004) hace un llamado para devolver la mirada hacia la cultura como vehículo del cambio, desde lo que él propone como desarrollo humano, dándole las capacidades, herramientas y facilidades, a las comunitarias y los comunitarios, de construirlo en atención a sus necesidades. La promueven como "(...) una fuerza viable para la sobrevivencia humana y como la fuente de desarrollo que continuamente se recrea a sí misma (...)" (2004, p. 152). Este mismo autor propone formas novedosas y creativas para originar desarrollo desde las comunidades. Al respecto expresa: "(...) Hay desarrollo donde hay crecimiento (...)" (2004, p. 280). Si amalgamamos los conceptos desarrollados a lo largo del ensayo, se visualiza la necesidad de promover, construir o instaurar espacios, desde la educación formal o no formal, que utilicen la expresión artística para la apropiación de las identidades y que faciliten, a su vez, la creación de un desarrollo autónomo y sustentable desde lo humanístico.

Nerfin, citado por Carmen (2004), establece que para que el desarrollo sea humanizante, debe generar niveles crecientes de autodependencia y sustentabilidad, en armonía con la naturaleza y la tecnología. "(...) Existe desarrollo donde la gente y sus 
comunidades - cualquiera sea el lugar y la duración- actúan como sujetos y no reciben una acción como objetos; afirman su autonomía, autodependencia y autoconfianza, cuando preparan y llevan a cabo sus proyectos (...) (Nerfin, 1981, p. 73)" (Carmen 2004, p. 280).

Las comunidades rurales están urgidas de espacios educativos que acorten las brechas existentes con la urbanidad, pero que establezcan, en sus mesas, como prioritario el tema de arraigo cultural de apropiación de las identidades de fortalecimiento del tejido social, para que las comunitarias y los comunitarios vean, en la educación, el aliciente para el desarrollo, un desarrollo construido desde sus necesidades, desde sus realidades.

Los desafíos de la educación en general y de la educación rural en particular son grandes, si los propósitos son la búsqueda de sociedades más solidarias, reflexivas, creativas y comprometidas con el devenir cultural y ambiental que les atañe. En esta concepción, la educación constituye la puerta de acceso a mejorar la calidad de vida. Desde Guatemala hasta Panamá, las reformas educativas se presentan como una oportunidad que debe responder a los requerimientos planteados por la globalización económica y cultural y enfocarse en la competitividad y equidad. Para lograr estos objetivos, en la región centroamericana se invierte, con apoyo de recursos externos, en adecuación curricular y capacitación de los recursos humanos (Aguilar, Cerdas y Céspedes, 2003, p. 56). Desde perspectivas humanizadoras y espontáneas, la expresión artística podría ayudar en la construcción de objetivos pragmáticos que generen, en las niñas y los niños, en las personas adolescentes y adultas mayores, la construcción y aceptación de sus realidades para potenciar las identidades culturales, individuales y colectivas.

Así como en Kenia y Brasil, en Costa Rica, en El Salvador y otros países de la región, proyectos liderados por los Ministerios de cultura y ONG's como Proarte, Casamarilla y Caja Lúdica son atrayentes y demuestran que se puede alfabetizar desde la esperanza, visualizando la alfabetización desde los lentes constructores de lectura y escritura de nuestro mundo, utilizando cualesquiera elementos o herramientas que potenciabilicen la expresión de ideas, sentimientos y aprendizajes. "(...) El alfabetizado se reencuentra en él, reencontrándose con los otros y en los otros, compañeros de su pequeño 'círculo de cultura"' (Freire, 2005, p. 14). Sin embargo, desde esta perspectiva, en Centroamérica, en las zonas rurales, es necesario proponer más espacios de expresión artística que fortalezcan la construcción de las identidades y la adquisición de competencias necesarias y que permitan la formación integral de los individuos.

Según Blanco y Cusato (2010), "América Latina es la región más inequitativa del mundo, en ella persisten la pobreza y la desigualdad distributiva que han dificultado la constitución de sociedades más integradas (...)" (p. 1). Centroamérica no se queda atrás, razón por la cual desde la educación, ya sea formal o no formal, es preciso realizar bosquejos de sitios de aprendiencia, en los cuales sus participantes potencialicen las aptitudes específicas que les permitan afianzar las identidades individuales y colectivas que dinamicen el acceso y achiquen las brechas económicas, sociales, educativas y culturales. Las zonas rurales de la región, se convirtieron en espacios físicos, geográficos de evidente abandono político, económico y sociocultural. 
Plantea Angulo, Morera y Torres que "(...) son las comunidades rurales las que presentan el menor índice de desarrollo social (...)” (2010, p. 3). “La calidad de la enseñanza y el aprendizaje está también asociada al contexto socioeconómico, las tradiciones e ideología de las y los [sic] participantes en el acto educativo y las políticas públicas que regulan el sistema" (Angulo, et al., 2010, p. 3). Razón de más, para que, desde la expresión artística, la educación genere espacios de representación de los saberes y sentires en miras de fortalecer el tejido social de las comunidades rurales.

La educación rural, tanto a nivel interno como a nivel de la región centroamericana, toma un matiz muy diferente de un contexto a otro, debido a los factores del entorno que en ella intervienen, tales como, ubicación geográfica, historia política, conflictos internos de carácter bélico, migración hacia las zonas rurales, entre otros. De acuerdo con el Dr. Jan Ooijens, mencionado por Aguilar, Cerdas y Céspedes, se experimentan tiempos de globalización que no benefician de igual forma a todos los habitantes de las zonas rurales. En la región centroamericana, este fenómeno se agudiza, pues la población de sus zonas rurales presenta características tales como exclusión social, pobreza extrema, desempleo, subempleo, incremento de personas analfabetas y de niños y jóvenes sin acceso al sistema de educción formal (2003). Con todas estas características, la población rural centroamericana se vuelve vulnerable a los embates de los cambios de un desarrollo globalizado.

El medio rural ha sufrido transformaciones en los niveles social, cultural, económico y político. Aguilar, Cerdas y Céspedes (2003) así lo afirman: "La cultura rural se ha tenido que adaptar, creando las condiciones para el desarrollo tecnológico de sus pueblos, ya sea prácticas de cultivo, hábitos de vida, tipo de trabajo, seguridad, tenencia de la tierra, relaciones de producción y consumo y visión del mundo." (2003, p. 40). Por tanto, se centra la esperanza del cambio en una educación que contemple, en su totalidad, al ser en formación, desde sus inicios de vida y durante su transcurso. Esta debe ser una educación basada en la expresión artística, que beneficie integralmente a los niños, niñas, y demás población rural: una educación centralizada en la expresión de sentires; que le permita a los seres humanos socializar, dialogar, tomar acuerdos en los que se valoren a sí mismos y a la otredad, guiados por el vehículo del respeto y nunca por el tren de la sumisión, con equidad e igualdad.

La educación es herramienta domesticadora o humanizadora; dependiendo del cristal con que se maneje. Se plantea, entonces, el reto de asumir los compromisos que la educación y las comunidades rurales nos delegan. Resulta fácil adquirir la homogenización de la cultura y adoptar identidades hegemónicas sin criterio alguno; pero si por el contrario, se utiliza la educación como instrumento liberador, de inmediato se encienden las llamas de la conciencia crítica y analítica en búsqueda de mejores sociedades, donde se armonicen y humanicen los espacios, se involucre la expresión de sentires desde las artes y se tome en cuenta la relación del ser humano y el medio cultural y ambiental en el que está inmerso. 


\section{Referencias}

Abad, J. (2006). Usos y funciones de las artes en la educación y el desarrollo humano. En L. Jiménez, I. Aguirre y L. G. Pimentel (Coords.), Educación artística, cultura y ciudadanía (pp. 17-23). España: OEl y Santillana. Recuperado de http://www.oei.es/metas2021/EDART2.pdf

Aguilar, M., Cerdas, Y. y Céspedes, E. (2003). Un acercamiento a la educación general básica de las zonas rurales de seis países centroamericanos. Heredia: Fundación Universidad Nacional.

Angulo, L., Morera, D. y Torres, N. (junio, 2010). Modelo y práctica pedagógica en la escuela rural unidocente y multigrado centroamericana: condiciones, limitaciones y potencialidades. Patria Grande. Revista Centroamericana de Educación 1(1), 1-13. Recuperado de http:// www.ceducar.info/OJS

Bayardo, R. (2009). Prólogo. En J. L. Mariscal (Comp.), Educación gestión cultural. Experiencias de acciones culturales en prácticas educativas (pp. 11-13). México: UDGvirtual. Recuperado de http://es.scribd.com/doc/44002395/Educacion-y-gestion-cultural-experiencias-deacciones-culturales-en-practicas-educativas

Blanco, R. y Cusato, S. (2010). Desigualdades educativas en América Latina: Todos somos responsables. Santiago de Chile: UNESCO. Recuperado de http://www.red-ler.org/ desigualdades educativas america latina.pdf

Brito, Z. (2008). Educación popular, cultura e identidad desde la perspectiva de Paulo Freire. En M. Gadotti, M. V. Gómez, J. Mafra y A. Fernandes (Comps.), Paulo Freire. Contribuciones para la pedagogía (pp. 16-30). Buenos Aires: CLACSO. Recuperado de http://es.scribd.com/ doc/25492640/Freire-Contribuciones-para-la-pedagogia

Carmen, R. (2004). Desarrollo autónomo: Humanización del paisaje: Una incursión en el pensamiento y la práctica radical. San José, Costa Rica: EUNA.

Castro, Y. (octubre, 2007). La educación para el desarrollo humano en un mundo globalizado. Revista Tendencia \& Retos, 12, 157-175. Recuperado de http://www.ts.ucr.ac.cr/binarios/ tendencias/rev-co-tendencias-12-10.pdf

Freire, P. (1993). Pedagogía de la esperanza. Un reencuentro con la pedagogía del opimido. Siglo XXI editores. Recuperado de http://archivosociologico.files.wordpress.com/2011/03/ freire-paulo-e28093-pedagogc3ada-de-la-esperanza.pdf

Freire, P. (2005). Pedagogía del oprimido (2a ed.). México: Siglo XXI Editores.

Harada, M. J., Pedroso, G. C. y Pereira, S. R. (2010). O teatro como estratégia para a construção da paz [El Teatro como estrategia para la construcción de la paz]. Acta Paulista de Enfermagem, 23(3), 429-432. Recuperado de http://www.scielo.br/pdf/ape/v23n3/en v23n3a19.pdf 
URL: http://www.una.ac.cr/educare

Jiménez, L., Aguirre, I. y Pimentel, L. G. (2006). Introducción. En L. Jiménez, I. Aguirre y L. G. Pimentel (Coords.), Educación artística, cultura y ciudadanía (pp. 17-23). España: OEl y Santillana. Recuperado de http://www.oei.es/metas2021/EDART2.pdf

Lagos, I., González, A. V. y Cárdenas, E. (2009). Expresión artística y cultura cotidiana en el libro: "Cuentos de Callaqui" Rexe. Revista de estudios y experincias en educación, 8(16), $25-37$. Recuperado de http://redalyc.uaemex.mx/redalyc/pdf/2431/Resumenes/243116384002 Resumen 1.pdf

Marchesi, Á. (2006). Preámbulo. En L. Jiménez, I. Aguirre y L. G. Pimentel (Coords.), Educación artística, cultura y ciudadanía (pp. 7-9). España: OEl y Santillana. Recuperado de http:// www.oei.es/metas2021/EDART2.pdf

Mariscal, J. L. (2009). Cruces e interacciones entre educación y gestión cultural (Introducción). En J. L. Mariscal (Comp.), Educación gestión cultural. Experiencias de acciones culturales en prácticas educativas (pp. 17-47). México: UDGvirtual. Recuperado de http://es.scribd. com/doc/44002395/Educacion-y-gestion-cultural-experiencias-de-acciones-culturalesen-practicas-educativas

Mollins, P., Castillo, P. y Silber, E. (2006). Cómo enseñar con proyectos integradores en la educación primaria. Argentina: Círculo Latino Austral.

Piñon, F. (2005). Presentación. IBEFORMAT, OEI y UNESCO (Autores), Formación en gestión cultural y políticas culturales. Directorio Iberoamericano de Centros de Formación. América Latina, Caribe, España y Portugal (pp. 11-12). París: UNESCO.Recuperado de http:// unesdoc.unesco.org/images/0013/001386/138686s.pdf

Reina, F. M. y Romero, J. (mayo, 2009). Expresión artística en situación extrema: Friedl DickerBrandeis y los niños de Terezín. Red Visión, 9 y 10, 1-7. Recuperado de http://www. redvisual.net/pdf/9-10/terezin.pdf

Rivas, P. (2008). El desarrollo en el vértice de la gestión cultural. Gestión Cultural, 1-24. Quito: Ministerio de Cultura de Ecuador. Recuperado de http://www.redinterlocal.org/IMG/ pdf desarrollo gestion cultural.pdf

Cómo citar este artículo, según APA:

Álvarez, S. y Domínguez, M. (2012). La expresión artística: Otro desafío para la educación rural. Revista Electrónica Educare, 16(3), 115-126. Consultado de http://www.revistas.una.ac.cr/index.php/EDUCARE/issue/current

Nota: Para citar este artículo en otros sistemas puede consultar el hipervínculo "Como citar el artículo" en la barra derecha de nuestro sitio web:

http://www.revistas.una.ac.cr/index.php/EDUCARE/index 